\title{
A CANONICAL FORM FOR A CLASS OF ORDINARY DIFFERENTIAL OPERATORS
}

\author{
HAROLD E. BENZINGER
}

\begin{abstract}
A canonical form is developed for each member of a large class of $n$th order linear ordinary differential operators, acting on $L^{P}(0,1)$. This canonical form shows how the Fourier-like behavior of eigenfunction expansions is related to structural properties of the operators.
\end{abstract}

1. Introduction. It is known [1]-[4], [8] that for a large class of ordinary differential operators corresponding to two-point boundary value problems, the convergence properties of the resulting eigenfunction expansions are the same as the convergence properties of ordinary Fourier series, with respect to both pointwise convergence and convergence in the norm of $L^{p}=L^{p}(0,1)$, $1<p<\infty$. In this paper we show that these properties are equivalent to the existence of a canonical form, relating such operators to a simple one. To be more specific, let $T$ denote an $n$th order differential operator which is Birkhoff regular with simple spectrum (see the Introduction to [3]), defined on a domain $\mathcal{D}(T)$ in $L^{p}$. Let $D$ denote the special case $D u=u^{(1)}$, where $\mathcal{D}(D)$ consists of all $u$ in $L^{p}$ such that $u$ is absolutely continuous, $u^{(1)}$ is in $L^{p}$, and $u(0)-u(1)$ $=0$. For $D$, the eigenfunctions are

$$
\varphi_{k}(x)=\exp (2 k \pi i x), \quad k=0, \pm 1, \ldots,
$$

and thus the resulting eigenfunction expansion for $u$ in $L^{p}$ coincides with the usual Fourier series expansion of $u$.

Theorem 1.1. Given $T$ and $p$, there is a bicontinuous linear map $A: L^{p} \rightarrow L^{p}$, and a multiplier transform $M$ on $L^{p}$, such that

$$
T=A(D+M)^{n} A^{-1} \text {. }
$$

Recall that a map $A$ is bicontinuous if $A$ and $A^{-1}$ are everywhere defined and continuous, and a bounded linear transformation $M$ on $L^{p}$ is a multiplier transform if it can be represented by

$$
M u=\sum_{-\infty}^{\infty} \alpha_{k}\left(u, \varphi_{k}\right) \varphi_{k}
$$

for some complex sequence $\left\{\alpha_{k}\right\} \cdot\left(\alpha_{k}=O\left(|k|^{1 / 2}\right)\right.$ is a sufficient condition that the series in (1.3) defines a multipler transform [5, p. 231].)

Received by the editors December 30, 1975.

AMS (MOS) subject classifications (1970). Primary 34B25.

Key words and phrases. Eigenfunction expansions.

- American Mathematical Society 1977 
The equation (1.2) contains all basic information about $T$. Since on $\mathscr{D}(D)$ we have

$$
(D+M) u=\sum_{-\infty}^{\infty}\left(2 k \pi i+\alpha_{k}\right)\left(u, \varphi_{k}\right) \varphi_{k},
$$

we see that the spectrum of $(D+M)^{n}$, and therefore, $T$, is the set

$$
\left\{\left(2 k \pi i+\alpha_{k}\right)^{n}: k=0, \pm 1, \ldots\right\} \text {. }
$$

Since the eigenfunctions of $D+M$ are the functions $\varphi_{k}, k=0, \pm 1, \ldots$, we see that the eigenfunctions of $T$ are the functions $u_{k}=A \varphi_{k}$. The pointwise and norm behavior of the eigenfunction expansions corresponding to $T$ can be deduced from the behavior of Fourier expansions, and properties of $A$ [3].

2. Preliminary results. Here we collect some facts about Fourier series and about the eigenfunctions of Birkhoff regular problems with simple spectrum. For fixed $p, 1<p<\infty,\left\{\varphi_{k}\right\}$ is a (Schauder) basis for $L^{p}[6$, p. 50]. It is easily seen that for $\left\{\alpha_{k}\right\}$ to define a multiplier transform as in (1.3), it is necessary that $\left\{\alpha_{k}\right\}$ be in $l^{\infty}$. We shall need that portion of the HausdorffYoung inequality [6, pp. 98-99] which states that if $f$ is in $L^{p}, 1 \leqslant p \leqslant 2$, then $\left\{\left(f, \varphi_{k}\right)\right\}$ is in $l^{q}(p q=p+q)$ and

$$
\left[\sum_{-\infty}^{\infty}\left|\left(f, \varphi_{k}\right)\right|^{q}\right]^{1 / q} \leqslant\|f\|_{p}
$$

Lemma 2.1. Let $\left\{\alpha_{k}\right\}$ be in $l^{\infty}$ and let $f$ be in $L^{p}, 1<p<\infty$. Let $n \geqslant 1$ and $j$ be integers. Let

$$
g_{j}=\sum_{-\infty}^{\infty} \alpha_{k}(2 k \pi i)^{j-n}\left(f, \varphi_{k}\right) \varphi_{k}
$$

Then for $j=0, \ldots, n-1$,

(1) the series (2.2) converges absolutely and uniformly on $0 \leqslant x \leqslant 1$,

(2) $g_{j}(x)=g_{0}^{(j)}(x)$,

(3) $g_{0}^{(j)}(0)-g_{0}^{(j)}(1)=0$.

If in addition $\left\{\alpha_{k}\right\}$ defines a multiplier transform on $L^{p}$,

(4) $g^{(n-1)}$ is absolutely continuous, and $g^{(n)}$ is in $L^{p}$.

Proof. Let $r=\min (p, 2)$, and define $s$ by $r s=r+s$. Then $1<r \leqslant 2$, and since $f$ is in $L^{p}$, we also have $f$ in $L^{r}$. Thus by the Hausdorff-Young inequality, $\left\{\left(f, \varphi_{k}\right)\right\}$ is in $l^{s}$. Since $n-j \geqslant 1$, we see that $\left\{(2 k \pi i)^{j-n}\right\}$ is in $l^{r}$. Thus since $\left|\alpha_{k}\right| \leqslant \mu$ for some $\mu>0$, we have

$$
\sum_{-\infty}^{\infty}\left|\alpha_{k}(2 k \pi i)^{j-n}\left(f, \varphi_{k}\right) \varphi_{k}(x)\right| \leqslant \mu\left[\sum_{-\infty}^{\infty}|2 k \pi i|^{(j-n) r}\right]^{1 / r}\|f\|_{r} .
$$

This establishes (1). Also, we see that for $j=0, \ldots, n-2$, the series for $g_{j}$ can be formally differentiated, producing a uniformly convergent series. This establishes (2), and also (3), since $\varphi_{k}(0)-\varphi_{k}(1)=0$. To establish (4), let 


$$
G=\sum_{-\infty}^{\infty} \alpha_{k}\left(f, \varphi_{k}\right) \varphi_{k}
$$

This series converges in the norm of $L^{p}$, so term-by-term integration is permitted:

$$
\begin{aligned}
\int_{0}^{x} G(t) d t & =\sum_{-\infty}^{\infty} \alpha_{k}(2 k \pi i)^{-1}\left(f, \varphi_{k}\right)\left[\varphi_{k}(x)-1\right] \\
& =g^{(n-1)}(x)-\sum_{-\infty}^{\infty} \alpha_{k}(2 k \pi i)^{-1}\left(f, \varphi_{k}\right) .
\end{aligned}
$$

This last series is convergent, by property (1). Thus

$$
g^{(n-1)}(x)=K+\int_{0}^{x} G(t) d t .
$$

This establishes (4).

REMARK. If in (2.2) we write

$$
g_{j}=\sum_{1}^{\infty} \alpha_{k}(2 k \pi i)^{j-n}\left(f, \varphi_{k}\right) e_{k},
$$

where $e_{k}(x)=\exp (a k x)$ and $a$ is a complex number with real part $\leqslant 0$, then properties (1), (2) are still valid.

Let $\mathcal{D}\left(D^{n}\right)$ denote those $u$ in $L^{p}$ such that $u^{(n-1)}$ is absolutely continuous, $u^{(n)}$ is in $L^{p}$, and $u^{(j)}(0)-u^{(j)}(1)=0, j=0, \ldots, n-1$. Then $D^{n}$ is the operator defined on $\mathscr{D}\left(D^{n}\right)$ by $D^{n} u=u^{(n)}$.

LEMMA 2.2. If $u$ is in $\mathscr{D}\left(D^{n}\right)$ and $M$ is a multiplier transform, then $M u$ is in OD $\left(D^{n}\right)$.

Proof. Using the boundary conditions and integration by parts, we have

$$
\left(D^{n} u, \varphi_{k}\right)=(2 k \pi i)^{n-j}\left(D^{j} u, \varphi_{k}\right), \quad j=0, \ldots, n-1 .
$$

Thus in the norm of $L^{p}$,

$$
M u=\sum_{-\infty}^{\infty} \alpha_{k}(2 k \pi i)^{-n}\left(D^{n} u, \varphi_{k}\right) \varphi_{k}
$$

and Lemma 2.1 can be applied. (Note that $\left(D^{n} u, \varphi_{0}\right)=0$.)

The general two-point boundary value problem can be written as

$$
\tau(u)=-\rho^{n} u, \quad U(u)=M \tilde{u}(0)+N \tilde{u}(1)=0,
$$

where for suitable functions $u$,

$$
\tau(u)=u^{(n)}+a_{n-2}(x) u^{(n-2)}+\cdots+a_{0}(x) u,
$$

with $a_{j}(x)$ in $L^{\infty}, M, N$ are $n \times n$ matrices of complex constants, and $\tilde{u}(x)$ is the transpose of $\left[u(x), \ldots, u^{(n-1)}(x)\right]$. To specify an operator $T$ on $L^{p}$, we restrict consideration to $\mathscr{D}(T)$, which consists of all $u$ in $L^{p}$ such that $u^{(n-1)}$ is absolutely continuous, $u^{(n)}$ is in $L^{p}$, and $M \tilde{u}(0)+N \tilde{u}(1)=0$. We shall also denote this operator by $(\tau, U)$.

In [3], it was proved that if (2.4) is Birkhoff regular with simple spectrum, 
then (in the notation used in [3]), the eigenfunctions $u_{k}(x)$ satisfy the asymptotic relations

$$
\begin{array}{ll}
u_{k}(x)=X_{1}(x) \varphi_{k}(x)\langle 1\rangle+X_{2}(x) \varphi_{-k}(x)\langle 1\rangle+F_{k}(x), & k \rightarrow+\infty \\
u_{k}(x)=X_{3}(x) \varphi_{k}(x)\langle 1\rangle+X_{4}(x) \varphi_{-k}(x)\langle 1\rangle+F_{k}(x), & k \rightarrow-\infty
\end{array}
$$

It is necessary to obtain similar estimates for $u_{k}^{(j)}(x), j=0, \ldots, n$. Starting from equation (13) in [7, p. 65], we see that the $j$ th derivative of the function $y$ given there can be obtained by differentiating the first row of the given determinant $j$ times. Then using the arguments in Theorems 5.2, 5.3 of [3], we have

LEMMA 2.3. If $(\tau, U)$ is any Birkhoff regular problem with simple spectrum, then the eigenfunctions $u_{k}(x)$ satisfy

$$
u_{k}^{(j)}(x)=(2 k \pi i)^{j} u_{k j}(x), \quad j=0, \ldots, n,
$$

where

$$
u_{k j}(x)=X_{1 j}(x) \varphi_{k}(x)\langle 1\rangle+X_{2 j}(x) \varphi_{-k}(x)\langle 1\rangle+F_{k j}(x)
$$

as $k \rightarrow+\infty$, and similarly as $k \rightarrow-\infty$.

LEMMA 2.4. If $u$ is in $\mathscr{D}(D)$, and if $M$ is a multiplier transform, then $D M u=M D u$.

Proof. By Lemma 2.2, $M u$ is in $\mathscr{D}(D)$. Then

$$
\begin{aligned}
D M u & =\sum_{-\infty}^{\infty} 2 k \pi i\left(M u, \varphi_{k}\right) \varphi_{k}=\sum_{-\infty}^{\infty} 2 k \pi i \alpha_{k}\left(u, \varphi_{k}\right) \varphi_{k} \\
& =\sum_{-\infty}^{\infty} \alpha_{k}\left(D u, \varphi_{k}\right) \varphi_{k}=M D u .
\end{aligned}
$$

Thus the expression $(D+M)^{n}$ can be expanded using the binomial theorem.

The sequence $\alpha_{k}=\operatorname{sgn} k$ is a multiplier sequence on $L^{p}, 1<p<\infty$, since these spaces admit conjugation [6, pp. 48,68]. Consequently any sequence

$$
\alpha_{k}=\mu_{1}, \quad k \rightarrow+\infty, \quad \alpha_{k}=\mu_{2}, \quad k \rightarrow-\infty,
$$

where $\mu_{1}, \mu_{2}$ are constants, is a multiplier sequence.

3. The main result. Let $\left\{\varphi_{k}\right\}$ be as defined in (1.2), and let $\left\{u_{k}\right\}$ be the sequence of eigenfunctions for a given $n$th order Birkhoff regular operator $T$ with simple spectrum. For fixed $p, 1<p<\infty$, let $A: L^{p} \rightarrow L^{p}$ be the bounded [3, Theorem 4.1] linear operator defined by

$$
A u=\sum_{-\infty}^{\infty}\left(u, \varphi_{k}\right) u_{k} .
$$

Clearly [3, Theorem 2.1] the inverse is

$$
A^{-1} u=\sum_{-\infty}^{\infty}\left(u, v_{k}\right) \varphi_{k}
$$


where $\left\{v_{k}\right\}$ denotes the sequence of eigenfunctions of $T^{*}$, normalized so that $\left(u_{k}, v_{j}\right)=\delta_{k j}$. Then the $v_{j}$ 's satisfy asymptotic relations of the form (2.6) [3, Theorem 5.4], and in particular, if $u$ is in $L^{p}, 1 \leqslant p \leqslant 2$, then $\left\{\left(u, v_{k}\right)\right\}$ is in $l^{q}, p q=p+q$, and $[3$, Theorem 3.2],

$$
\left[\sum_{-\infty}^{\infty}\left|\left(u, v_{k}\right)\right|^{q}\right]^{1 / q} \leqslant K_{p}\|u\|_{p} .
$$

THEOREM 3.1. $\mathscr{Q}(T)=A^{\mathscr{D}}\left(D^{n}\right)$.

Proof. First we show that $A^{\mathscr{D}}\left(D^{n}\right) \subset \mathscr{D}(T)$. For $u$ in $\mathscr{D}\left(D^{n}\right)$, we have (2.3), and thus

$$
A u=\sum_{-\infty}^{\infty}(2 k \pi i)^{-n}\left(D^{n} u, \varphi_{k}\right) u_{k}
$$

and by (2.7), (2.8), the formal derivatives of the above series are

$$
\sum_{-\infty}^{\infty}(2 k \pi i)^{j-n}\left(D^{n} u, \varphi_{k}\right) u_{k j}
$$

By application of Lemma 2.1 and the remark following it, we see that this series is uniformly convergent for $0 \leqslant x \leqslant 1, j=0, \ldots, n-1$. Thus these series represent $(A u)^{(j)}$ for $j=0, \ldots, n-1$, and since the $u_{k}$ 's satisfy the boundary conditions, so does $A u$. Clearly $A u$ is in $L^{p}$. To show that $(A u)^{(n-1)}$ is absolutely continuous, and $(A u)^{(n)}$ is in $L^{p}$, consider

$$
w=\sum_{-\infty}^{\infty}(2 k \pi i)^{-n}\left(D^{n} u, \varphi_{k}\right) D^{n} u_{k}
$$

Using Lemma 2.3, we see that the series in (3.6) converges in the norm of $L^{p}$, and it can be integrated term-by-term:

$$
\begin{aligned}
W(x)= & \int_{0}^{x} w(t) d t=\sum_{-\infty}^{\infty}(2 k \pi i)^{-1}\left(D^{n} u, \varphi_{k}\right) u_{k n-1}(x) \\
& -\sum_{-\infty}^{\infty}(2 k \pi i)^{-1}\left(D^{n} u, \varphi_{k}\right) u_{k n-1}(0)
\end{aligned}
$$

where this last series is convergent by the remark following Lemma 2.1. Thus

$$
(A u)^{(n-1)}(x)=K+\int_{0}^{x} w(t) d t
$$

Next we show that $A^{-1} \mathscr{D}(T) \subset \mathscr{D}\left(D^{n}\right)$. Let $u$ be in $\mathscr{D}(T)$ and let $A^{-1} u$ be given by (3.2). Then using (3.3), the above arguments can be repeated (in slightly simpler form).

Proof of TheOrem 1.1. Let $\lambda_{k}=-\rho_{k}^{n}, k=0, \pm 1, \ldots$, denote the sequence of eigenvalues of $T$. Using the asymptotic estimates from [3, Theorem 5.1], we see that 


$$
\begin{array}{ll}
\lambda_{k}=-\left[2 k \pi i \omega_{\mu}^{-1}+\mu_{1}+\beta_{k}\right]^{n}, & k \rightarrow+\infty, \\
\lambda_{k}=-\left[2 k \pi i \omega_{\mu}^{-1}+\mu_{2}+\beta_{k}\right]^{n}, & k \rightarrow-\infty,
\end{array}
$$

Where $\mu_{1}, \mu_{2}$ are constants, $\omega_{\mu}$ is a $n$th root of -1 , and $\beta_{k}=O\left(k^{-1}\right)$. Note that the sequence $\left\{\alpha_{k}\right\}$ defined by

$$
\begin{array}{ll}
\alpha_{k}=\omega_{\mu}\left[\mu_{1}+\beta_{k}\right], & k \rightarrow+\infty, \\
\alpha_{k}=\omega_{\mu}\left[\mu_{2}+\beta_{k}\right], & k \rightarrow-\infty,
\end{array}
$$

is a multiplier sequence. Let $u$ be in $\mathscr{D}(T)$. Then since $\left\{u_{k}\right\}$ is a basis for $L^{p}$ equivalent to $\left\{\varphi_{k}\right\}[3$, Theorem 5.3], we have

$$
\begin{aligned}
T u & =\sum_{-\infty}^{\infty}\left(T u, v_{k}\right) u_{k}=A \sum_{-\infty}^{\infty}\left(u, T^{*} v_{k}\right) \varphi_{k} \\
& =A \sum_{-\infty}^{\infty} \lambda_{k}\left(u, A^{-1 *} \varphi_{k}\right) \varphi_{k}=A \sum_{-\infty}^{\infty}(-1)\left(\omega_{\mu}^{-n}\right)\left[2 k \pi i+\alpha_{k}\right]^{n}\left(A^{-1} u, \varphi_{k}\right) \varphi_{k} \\
& =A \sum_{-\infty}^{\infty}\left[2 k \pi i+\alpha_{k}\right]^{n}\left(A^{-1} u, \varphi_{k}\right) \varphi_{k} \\
& =A \sum_{-\infty}^{\infty}\left(\sum_{r=0}^{n}\left(\begin{array}{l}
n \\
r
\end{array}\right)(2 k \pi i)^{r} \alpha_{k}^{n-r}\right)\left(A^{-1} u, \varphi_{k}\right) \varphi_{k} \\
& =A \sum_{r=0}^{n}\left(\begin{array}{l}
n \\
r
\end{array}\right) \sum_{-\infty}^{\infty}(2 k \pi i)^{r} \alpha_{k}^{n-r}\left(A^{-1} u, \varphi_{k}\right) \varphi_{k} \\
& =A \sum_{r=0}^{n}\left(\begin{array}{l}
n \\
r
\end{array}\right) D^{r} M^{n-r} A^{-1} u=A(D+M)^{n} A^{-1} u .
\end{aligned}
$$

\section{REFERENCES}

1. H. E. Benzinger, Green's function for ordinary differential operators, J. Differential Equations 7 (1970), 478-496. MR 41 \# 7189.

2. $\_$The $L^{p}$ behavior of eigenfunction expansions, Trans. Amer. Math. Soc. 174 (1972), 333-344 MR 48 \#6531.

3. Pointwise and norm convergence of a class of biorthogonal expansions, Trans. Amer. Math. Soc. (to appear).

4. G. D. Birkhoff, Boundary value and expansion problems of ordinary linear differential equations, Trans. Amer. Math. Soc. 9 (1908), 373-395.

5. I. I. Hirschman, Jr., On multiplier transformations, Duke Math. J. 26 (1959), 221-242. MR 21 $\# 3721$.

6. Y. Katznelson, An introduction to harmonic analysis, Wiley, New York, 1968. MR 40 \# 1734.

7. M. A. Naimark, Linear differential operators, GITTL, Moscow, 1954; German transl., Akademie-Verlag, Berlin, 1960. MR 16, 702.

8. M. H. Stone, A comparison of the series of Fourier and Birkhoff, Trans. Amer. Math. Soc. 28 (1926), 695-761.

Department of Mathematics, University of Illinois, Urbana, Illinois 61801 